\title{
Assessing the signatures imprinted by star-forming galaxies in the cosmic $\gamma$-ray background
}

\author{
Ellis R. Owen, ${ }^{a, b, *}$ Khee-Gan Lee ${ }^{c}$ and Albert K. H. Kong ${ }^{a}$ \\ ${ }^{a}$ Institute of Astronomy, National Tsing Hua University, Hsinchu, Taiwan (ROC) \\ ${ }^{b}$ Center for Informatics and Computation in Astronomy, National Tsing Hua University, \\ Hsinchu, Taiwan (ROC) \\ ${ }^{c}$ Kavli Institute for the Physics and Mathematics of the Universe, University of Tokyo, Kashiwa, Chiba \\ 277-8583, Japan \\ E-mail: erowen@gapp.nthu.edu.tw
}

In recent years, $\gamma$-ray emission has been detected from star-forming galaxies (SFGs) in the local universe, including M82, NGC 253, Arp 220 and M33. The bulk of this emission is thought to be of hadronic origin, arising from the interactions of cosmic rays (CRs) with the interstellar medium of their host galaxy. Distant SFGs are presumably also bright in $\gamma$-rays. Although they would not be resolvable as point sources, distant unresolved SFG populations contribute $\gamma$-rays to the extra-galactic $\gamma$-ray background (EGB). Despite the wealth of high-quality all-sky EGB data collected over more than a decade of operation with the Fermi-LAT $\gamma$-ray space telescope, the exact contribution of SFGs to the EGB remains unsettled. In this study, we model the $\gamma$-ray emission from SFG populations and demonstrate that such emission can be characterized by just a small number of physically-motivated parameters. We further show that source populations would leave anisotropic signatures in the EGB, which could be used to yield information about the underlying properties, dynamics and evolution of CR-rich SFGs.

$37^{\text {th }}$ International Cosmic Ray Conference (ICRC 2021)

July 12th - 23rd, 2021

Online - Berlin, Germany

\footnotetext{
*Presenter
} 


\section{Introduction}

Several nearby star-forming galaxies (SFGs) have been resolved in $\gamma$-rays [1], establishing such systems as a candidate source class for the extra-galactic $\gamma$-ray background (EGB). Their $\gamma$-ray emission is driven by an abundant reservoir of cosmic rays (CRs). These interact with interstellar gases to form pions $\left(\pi^{0}, \pi^{ \pm}\right)$, with $\gamma$-ray photons being released by $\pi^{0}$ decays. The CRs within these systems are presumably accelerated by diffusive shock acceleration processes, boosting low energy charged seed particles to relativistic energies, which would be operating in the abundance of violent shocked astrophysical environments that would emerge soon after the onset of star-formation.

The EGB can be decomposed into two components: (1) the $\gamma$-ray emission arising from resolved extra-galactic source populations, and (2) an isotropic component comprised of the accumulated emission from all unresolved $\gamma$-ray emitting sources beyond our Galaxy, extending as far as the observable Universe. The contribution to the EGB from source classes such as blazars and AGN has been well studied [e.g. 2], with many such sources having been resolved (either directly using $\gamma$-rays, or by association with observations at other wavelengths). However, the exact balance of sources forming the EGB has not yet been firmly established. While it has been argued that the majority of the EGB flux originates in unresolved AGN sources, including blazars [e.g. 3] and radio galaxies [e.g. 4], SFGs could still account for several tens of percent [5].

Recently, the SFG contribution to the EGB was modeled using a prototype approach [see 5], with $\gamma$-ray emission from source galaxies being weighted by their star-formation rates compared to the nearby starburst M82. Other studies developed physically-determined SFG template models [e.g. 6, which returned broadly similar EGB predictions as the M82 prototype method]. It was further considered [in 6] that spatial anisotropies would be imprinted into the $\gamma$-ray sky by a SFG population, and these were demonstrated to be sensitive to certain inherent properties of an source population of SFGs - in particular, the internal CR spectral index and the redshift distribution of the population. This work extends these earlier results, and further assesses the impact source galaxy properties would have in modifying the SFG contribution to the EGB and its imprinted spatial signatures.

\section{Emission of $\gamma$-rays from SFGs}

In SFGs, energetic hadronic CRs would predominantly interact through proton-proton ( $\mathrm{pp}$ ) pionproduction processes [7]. This arises above a threshold proton kinetic energy of $T_{\mathrm{p}}^{\text {th }}=0.28 \mathrm{GeV}$, with around $30 \%$ of pions produced being $\pi^{0}$, from which $\gamma$-ray production proceeds (with a branching ratio of $98.8 \%$ and timescale of $\sim 10^{-16} \mathrm{~s}$ ) as $\pi^{0} \rightarrow 2 \gamma$. The weak energy-dependence of the pp interaction cross-section and the $\pi^{0}$ formation multiplicity yields a $\gamma$-ray spectrum closely tracing the shape of the underlying CR proton spectrum driving the emission.

\subsection{CR spectrum and energy budget}

The internal CR spectrum of a SFG is typically well-described by a simple power-law. The spectral index $\Gamma$ has been found range from -1.9 to -2.3 in nearby SFGs detected in $\gamma$-rays [e.g. 1]. Here, we adopt a proton spectrum of $\Gamma=-2.1$, i.e. the mid-value of this range. The CR proton density within a SFG is estimated following the approach of [6]. This can be parameterised using just five quantities: the star-formation rate of the host galaxy, $\mathcal{R}_{\mathrm{SF}}$, the size of the nuclear starburst region, 
$R$, the $\mathrm{CR}$ spectral index, $\Gamma$, the maximum $\mathrm{CR}$ proton energy, $E_{\max }$ and an advective escape fraction $f_{\text {adv }}$, which is the fraction of CRs that would be removed from a SFG by advection in galactic outflows. Large-scale galactic outflows are common in distant, young SFGs [see, e.g. 8] and are driven by the confluence of feedback from the concentrated starburst episode arising in galactic cores. The impact of these outflows on the steady-state CR energy density in a galaxy can be substantial, reducing it by between a few and a few tens of percent [9]. We discuss our model for $f_{\text {adv }}$ in section 2.4. In the following calculations, we fix $R=0.1$ and $E_{\max }=50 \mathrm{PeV}$ [6]. $\mathcal{R}_{\mathrm{SF}}$ is specified by the adopted star-formation rate function (SFRF) for the population [10].

\subsection{Template emission model}

The volumetric rate at which pp interactions arise is given by $\dot{n}_{\mathrm{p} \pi}\left(\gamma_{\mathrm{p}}\right)=\left\langle n_{\mathrm{H}}\right\rangle n_{\mathrm{p}}\left(\gamma_{\mathrm{p}}\right) c \sigma_{\mathrm{p} \pi}\left(\gamma_{\mathrm{p}}\right)$ [6], where $\left\langle n_{\mathrm{H}}\right\rangle$ is the average ambient gas density within a SFG, $n_{\mathrm{p}}$ is the CR proton density (which we set as $1 \mathrm{~cm}^{-3}$ ), and $\sigma_{\mathrm{p} \pi}$ is the total inelastic pp interaction cross-section. This is well-parametrised by [11]. The production rate of $\gamma$-rays, which relies on the formation and subsequent decay of neutral pions, is described by the differential $\gamma$-ray inclusive cross section of the pp $\rightarrow \mathrm{pp} \pi^{0}$ interaction channel. This is also well-parametrised to an accuracy of better than 10 per cent by [11]. The emitted $\gamma$-rays can be attenuated by $\gamma \gamma$ pair-production between high-energy $\gamma$-rays and a low energy target photons provided by the CMB, starlight or dust-reprocessed starlight. This proceeds as $\gamma+\gamma \rightarrow e^{+}+e^{-}$at a rate $\dot{N}_{\gamma \gamma}$ determined by the spectral number density of target photons and the effective $\gamma \gamma$ interaction cross-section [see, e.g. 12]. We define the characteristic $\gamma$-ray path length in a radiation field as $\ell_{\gamma \gamma}\left(\epsilon_{\gamma}, x\right)=c / \dot{N}_{\gamma \gamma}\left(\epsilon_{\gamma}, x\right)$. This is the distance over which an interaction would typically arise under conditions specified at location $x$ [see 12], which can be used to quantify the $\gamma$-ray attenuation factor within a galaxy. When averaged through an extended SFG source (modeled as a uniformly attenuating sphere of radius $R$ ), this may be approximated by a characteristic attenuation factor specified by the size of the star-forming nucleus $R$ and the (energy-dependent) effective $\gamma$-ray path length as $\mathcal{A}(\zeta)=\exp \left(-\zeta^{2}\right)[6]$, where $\zeta=\left(R / \ell_{\gamma \gamma}\right)^{1 / 2}$.

We consider that $\gamma$-ray attenuation within a SFG is dominated by three radiation fields: (1) the cosmological microwave background (CMB); (2) the interstellar radiation field (ISRF) from stars, and (3) the re-processed ISRF by interstellar dust. These may each be approximated with a blackbody spectrum defined by their characteristic temperature. We follow the treatment in [6], and the attenuating effects of these radiation fields on the emitted spectrum are demonstrated in Figure 1 for a template galaxy example with $\mathcal{R}_{\mathrm{SF}}=10 \mathrm{M}_{\odot} \mathrm{yr}^{-1}, R=0.1 \mathrm{kpc}$ and located at a redshift of $z=2$.

\subsection{Galaxy clustering}

The spatial distribution of SFGs, described by their power spectrum $P_{\mathrm{g}}(k, z)$, is reflected in their emission imprinted into the EGB. The bias of galaxy population clustering compared to that of dark matter is typically studied observationally from their spatial distribution, with various source classes having been found to exhibit different clustering properties [e.g. see 13, which finds a different clustering bias for AGNs and SFGs against dark matter]. To model this bias, we assume linear clustering of SFGs, and adopt a galaxy clustering length of $r_{0}^{\mathrm{c}}=6.1 \mathrm{Mpc} h^{-1}$, and a power-law slope of the two-point correlation function of galaxies of $\iota=1.8$ [13]. 

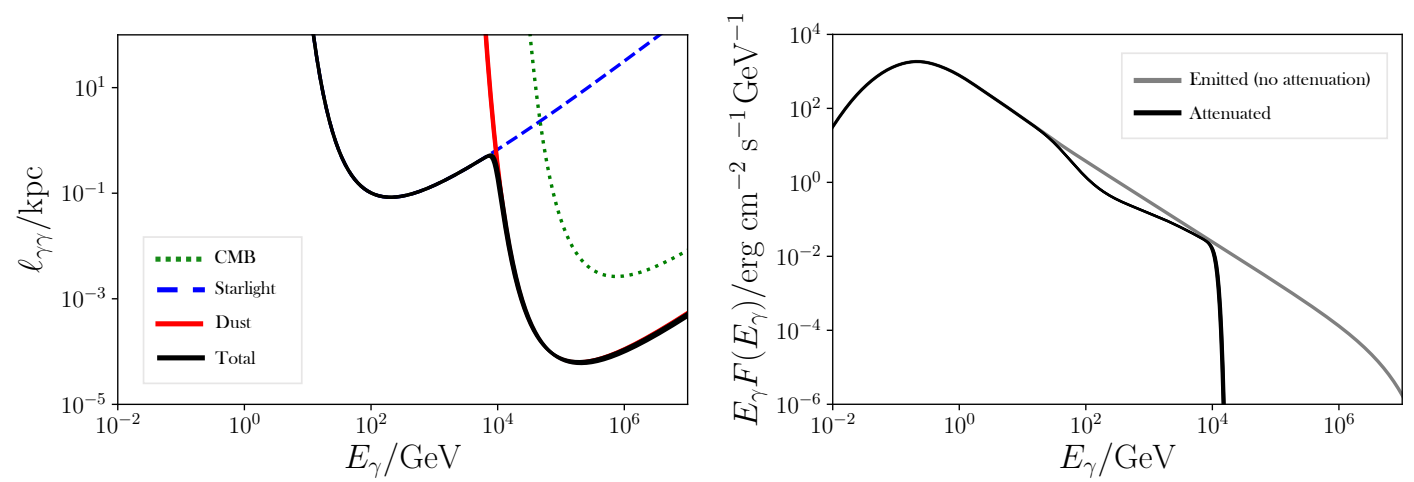

Figure 1: Adapted from [6], with $\mathcal{R}_{\mathrm{SF}}=10 \mathrm{M}_{\odot} \mathrm{yr}^{-1}, R=0.1 \mathrm{kpc}$ and at $z=2$. Left: Effective path lengths of $\gamma$-rays within a SFG model. Attenuation is strongly dominated by dust emission from $\sim 10 \mathrm{TeV}$. Right: $\gamma$-ray emission from the pp interaction is shown in grey. The black line shows the effective $\gamma$-ray emission spectrum emitted from the model SFG, accounting for $\gamma$-ray absorption within the SFG nucleus.

\subsection{Outflows and CR propagation in SFGs}

The impact of outflows, which are ubiquitous among SFGs, in removing CRs from a galaxy has been argued to be substantial [9], and this could have significant impacts for the resulting $\gamma$-ray emission from a source population. [6] accounted for this by simply introducing a CR reduction factor of 50\% uniformly across all SFGs. However, recent works have demonstrated that outflow velocities can evolve over redshift, particularly up to $z \sim 2$ [14], with additional dependencies on $\mathcal{R}_{\mathrm{SF}}$ and the stellar mass of a host galaxy. This would modify the $\mathrm{CR}$ containment fraction compared to that assumed in the earlier model of [6]. We introduce a simple CR containment model, where the CR spectral normalization is set by the advective loss fraction: ${ }^{1}$

$$
\left.f_{\mathrm{adv}}\left(E_{\mathrm{p}}\right) \approx\left\{\frac{V_{\mathrm{f}}(\ell) \tau_{\mathrm{adv}}^{-1}(\ell)}{\tau_{\mathrm{d}}^{-1}\left(E_{\mathrm{p}}\right)+\tau_{\mathrm{adv}}^{-1}(\ell)}\right\}\right|_{\ell=\ell_{0}} .
$$

Here, $V_{\mathrm{f}}(\ell)$ is the volume filling factor of an outflow as a function of height $\ell$ from the starburst nucleus, $\tau_{\mathrm{d}}\left(E_{\mathrm{p}}\right)=\ell^{2} / 4 D\left(E_{\mathrm{p}}\right)$ is the characteristic CR diffusion timescale, and $\tau_{\mathrm{adv}}=\ell / v(\ell) \sim \ell / v_{\infty}$ is the characteristic CR advection timescale in an outflow. $v\left(\ell_{0}\right) \approx v_{\infty}$ is the velocity of the outflow at some point that may be considered as the interstellar boundary, beyond which the flow is approximated to have reached its terminal velocity. We set this point as $\ell_{0}=1 \mathrm{kpc}$.

Some studies have considered that $V_{\mathrm{f}}$ may depend on redshift [15], however the exact dependency remains unclear and presumably would reflect the detailed physical configuration of an outflow and its immediate environment. As such, we follow [16], and adopt a value of 0.6, corresponding to the volume filling factor of the hot wind fluid at around $\ell_{0}=1 \mathrm{kpc}$. We only consider variation of the diffusion coefficient through its energy-dependence, which takes the parametric form $D\left(\gamma_{\mathrm{p}}\right)=D_{0}\left(r_{L}\left(\gamma_{\mathrm{p}},\langle|B|\rangle\right) \mid / r_{L, 0}\right)^{\varsigma}$, where $\langle|B|\rangle=|B|$ is the characteristic local magnetic field strength. The normalization value $D_{0}=3.0 \times 10^{28} \mathrm{~cm}^{2} \mathrm{~s}^{-1}$ is based on empirical measure-

\footnotetext{
${ }^{1}$ This approximation would not be valid at very high energies, for which faster CR diffusion would not effectively 'contain' CRs in a host galaxy/outflow system.
} 
ments of the diffusion of CRs in the Milky Way, and is appropriate for a $1 \mathrm{GeV}$ CR proton diffusing through a $5 \mu \mathrm{G}$ ISM magnetic field with gyro-radius $r_{L, 0}$.

If assuming the same volume filling factor $V_{\mathrm{f}}=0.6$, and that all SFGs are affected equally by outflows, the baseline model of [6] would correspond to an outflow velocity of $\sim 80 \mathrm{~km} \mathrm{~s}^{-1}$, i.e. significantly less than observationally determined ranges of values [e.g. 14]. Indeed, this choice was intended to indicate an upper estimate for the EGB intensity at $z=0$ but, if taken strictly, it would imply either substantially smaller filling factors were implicitly assumed, or only a subset of SFGs would host sufficiently strong outflows to advect CRs. In this work, we consider the impact a more physically-informed treatment of CR transport would have on the production of $\gamma$-rays in SFGs, accounting for the evolutionary trends of galactic outflow velocities over redshift described by [14], using the scaling relation

$$
v_{\infty}\left(z, M^{\star}\right) \approx 320\left(\frac{\mathrm{sSFR}}{0.316 \mathrm{Gyr}^{-1}}\right)^{\beta_{\mathrm{v}}(z)} \mathrm{km} \mathrm{s}^{-1},
$$

where sSFR $=\mathcal{R}_{\mathrm{SF}} / M_{\star}$ is the specific star-formation rate of a galaxy, and $\mathcal{R}_{\mathrm{SF}}$ is set by the redshift and stellar mass of a galaxy. We consider three forms for the function $\beta_{\mathrm{v}}(z)$, inspired by the best-fit values of [14], where Model 1 reflects their full sample of $z<0.2$ to $z=2.2$ galaxies with $\beta_{\mathrm{v}}(z)=0.46$; Model 2 reflects their restricted sample of galaxies up to $z=1.4$, where $\beta_{\mathrm{v}}(z)=0.58$; and Model 3 reflects the minimal redshift evolution scenario above $z=2$ [17] such that $\beta_{\mathrm{v}}(z)=0.58 \mathcal{H}(2-z) .^{2}$

\section{EGB model}

\subsection{Cosmological propagation of $\gamma$-rays}

Over cosmological distances, the radiative transfer equation for $\gamma$-rays, in terms of redshift, is:

$$
\frac{\mathrm{d} \mathcal{I}_{\gamma}}{\mathrm{d} z}=(1+z)\left[-\alpha_{\gamma \gamma} \mathcal{I}_{\gamma}+\frac{j_{\gamma}}{v^{3}}\right] \frac{\mathrm{d} s}{\mathrm{~d} z}
$$

where all quantities are Lorentz invariant, i.e. $I_{\gamma}=I_{\gamma} / v^{3}$ for $I_{\gamma}$ as the local 'proper' intensity (such that, in practice, co-moving absorption $\alpha_{\gamma \gamma}$ and emission $j_{\gamma}$ functions are used for the attenuation and emission of $\gamma$-rays respectively, and $v$ is co-moving frequency). $\mathrm{d} s / \mathrm{d} z$ is defined for a flat Friedmann-Robertson-Walker (FRW) Universe. We solve Equation 3 numerically over redshift to model the SFG contribution to the EGB spectrum at $z=0$, with the SFG source population (using [10]) distributed up to $z_{\max }=3 .^{3}$

Over cosmological distances, $\gamma$-rays are reprocessed by pair-production in the extra-galactic background light (EBL) and subsequent inverse-Compton scattering off CMB photons (the $\gamma$-ray cascade). The attenuating part of this process can be characterized by an optical depth:

$$
\tau_{\gamma \gamma}\left(z, \epsilon_{\gamma}\right) \equiv \int_{0}^{z} \alpha_{\gamma \gamma}\left(z^{\prime}, \epsilon_{\gamma}\right) \frac{\mathrm{d} s}{\mathrm{~d} z^{\prime}} \mathrm{d} z^{\prime} .
$$

\footnotetext{
${ }^{2} \mathcal{H}$ is the Heaviside step function.

${ }^{3}$ This range covers the peak of cosmic star-formation, and would presumably account for the majority of $\gamma$-ray emission from SFGs.
} 


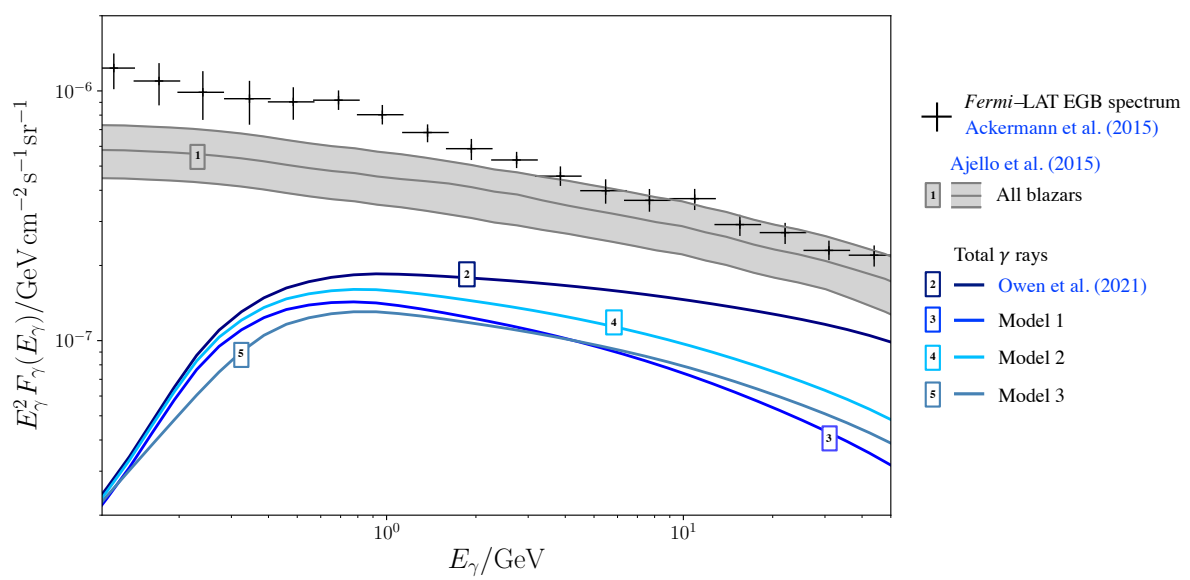

Figure 2: EGB spectrum from SFGs under the 3 outflow redshift-dependency models. While CR advection in outflows is important at lower energies, diffusive propagation starts to become more competitive at higher energies. For all 3 outflow models, the EGB flux is reduced at all energies compared to the results of Owen et al. 2021 [6], however this reduction is more substantial at higher energies. The band in grey, labeled as line 1, represents the 'all blazar' contribution of Ajello et al. 2015 [3], while the Fermi-LAT data points are from Ackermann et al. 2015 [19].

The associated cascade emission is computed assuming inverse-Compton scattering of pair-produced electrons dominates, and this is encoded by the term $j_{\gamma}$ together with direct emission from SFGs. If adopting the the semi-analytic model of [18], for which $\gamma$-ray optical depths for the EBL are provided between $1 \mathrm{GeV}$ and $45 \mathrm{TeV}$, and up to a redshift of $z=10$, the $\gamma$-ray absorption coefficient $\alpha_{\gamma \gamma}$ can be computed from the differential optical depth as a function of redshift.

We solve equation 3 for a fixed outflow loss fraction of 50\% (thus reproducing the result of [6]), and for the three outflow velocity models discussed in section 2.4. The resulting EGB spectrum is shown in Figure 2, which shows the choice of CR transport model to be important - particularly at higher energies, where diffusive CR propagation becomes more significant.

\subsection{EGB anisotropy}

The SFG power spectrum $P_{\mathrm{g}}(k, z)$ (see section 2.3) would imprint a spatial signature in the EGB, even though individual contributing sources would not typically be resolved. The distribution of spatial scales of this signature would depend on redshift $z$, being specified by $P_{\mathrm{g}}(k, z)$ and the strength of the contribution from sources at a particular epoch. The imprinted signatures could be accessed in the EGB using the intensity fluctuation power spectrum $C_{\ell}$, providing an alternative window to study EGB source populations and their variation over $z$.

With sufficiently detailed modeling of signatures, crucial insights about the CR activity in EGB source populations could be uncovered from anisotropies in the $\gamma$-ray sky. As a demonstration, Figure 3 shows how the EGB anisotropy signature differs between the four source models considered in this work. Notably, Model 3 (which introduces an additional redshift dependence compared to the other models) shows a different shape of EGB anisotropy signature, indicating how underlying differences in source populations and their evolution may be probed. Such signatures would be accessible in the near-future with Fermi-LAT and the up-coming Cherenkov Telescope Array [6]. 


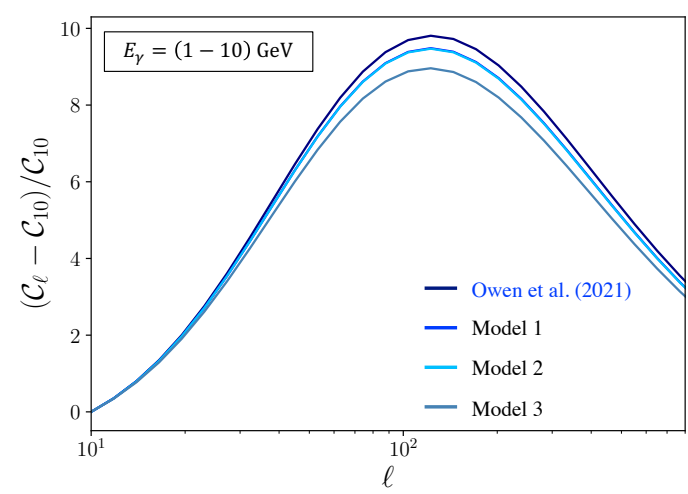

Figure 3: EGB intensity fluctuation power spectrum $C_{\ell}$, normalized to $C_{10}$, plotted against multipole $\ell$ in the energy band $E_{\gamma}=(1-10) \mathrm{GeV}$. This shows the result of Owen et al. 2021 [6], compared to the 3 outflow models, as labeled, which all show a slightly broader power spectrum. This is most noticeable in the case of Model 3 , where the broadening is stronger, particularly towards larger scales (smaller $\ell$ ).

\section{Summary and conclusions}

This work has shown how signatures are imprinted into the EGB by SFG populations, and that these can be modeled using a template approach, specified by just a small number of physically-motivated parameters. The resulting EGB spectrum was shown to be sensitive to source population properties, in particular CR transport processes in SFGs which must be carefully modeled in future work to robustly determine their exact EGB contributions. Information about the redshift distribution of EGB sources was shown to be accessible in the EGB intensity fluctuation power spectrum which, with detailed modeling, could be used to uncover the redshift distribution of contributing source populations and even reveal details about their evolutionary characteristics. This could have important applications in resolving CR activity in galaxies during the 'high noon' of cosmic star-formation.

\section{Acknowledgments}

This work used high-performance computing facilities operated by the Center for Informatics and Computation in Astronomy (CICA) at National Tsing Hua University (NTHU). This equipment was funded by the Ministry of Education of Taiwan and the Ministry of Science and Technology of Taiwan (MOST). ERO is supported by the Ministry of Education of Taiwan at CICA, NTHU. KGL acknowledges support from JSPS KAKENHI grants JP18H05868 and JP19K14755. AKHK acknowledges support from MOST (grant 109-2628-M-007-005-RSP).

\section{References}

[1] M. Ajello, M. Di Mauro et al., The $\gamma$-Ray Emission of Star-forming Galaxies, ApJ 894 (2020) 88 [2003.05493].

[2] A. Lamastra, N. Menci et al., Extragalactic gamma-ray background from AGN winds and star-forming galaxies in cosmological galaxy-formation models, A\&A 607 (2017) A18 [1709.03497].

[3] M. Ajello, D. Gasparrini et al., The Origin of the Extragalactic Gamma-Ray Background and Implications for Dark Matter Annihilation, ApJ 800 (2015) L27 [1501.05301]. 
[4] F.W. Stecker, C.R. Shrader et al., The Extragalactic Gamma-Ray Background from Core-dominated Radio Galaxies, ApJ 879 (2019) 68 [1903.06544].

[5] E. Peretti, P. Blasi et al., Contribution of starburst nuclei to the diffuse gamma-ray and neutrino flux, MNRAS 493 (2020) 5880 [1911.06163].

[6] E.R. Owen, K.-G. Lee et al., Characterizing the signatures of star-forming galaxies in the extragalactic $\gamma$-ray background, MNRAS 506 (2021) 52 [2106.07308].

[7] E.R. Owen, I.B. Jacobsen et al., Interactions between ultra-high-energy particles and protogalactic environments, MNRAS 481 (2018) 666 [1808.07837].

[8] B. Frye, T. Broadhurst et al., Spectral Evidence for Widespread Galaxy Outflows at $z>4$, ApJ 568 (2002) 558 [astro-ph/0112095].

[9] E. Peretti, P. Blasi et al., Cosmic ray transport and radiative processes in nuclei of starburst galaxies, MNRAS 487 (2019) 168 [1812.01996].

[10] A. Katsianis, G. Blanc et al., The evolution of the star formation rate function in the EAGLE simulations: a comparison with UV, IR and H $\alpha$ observations from $z \sim 8$ to $z \sim 0, M N R A S$ 472 (2017) 919 [1708.01913].

[11] E. Kafexhiu, F. Aharonian et al., Parametrization of gamma-ray production cross sections for $p$ p interactions in a broad proton energy range from the kinematic threshold to PeV energies, PhRvD 90 (2014) 123014 [1406.7369].

[12] R.J. Gould and G.P. Schréder, Pair Production in Photon-Photon Collisions, Physical Review 155 (1967) 1404.

[13] C.L. Hale, M.J. Jarvis et al., The clustering and bias of radio-selected AGN and star-forming galaxies in the COSMOS field, MNRAS 474 (2018) 4133 [1711.05201].

[14] Y. Sugahara, M. Ouchi et al., Evolution of Galactic Outflows at $z \sim 0-2$ Revealed with SDSS, DEEP2, and Keck Spectra, ApJ 850 (2017) 51 [1703.01885].

[15] S. Bertone, F. Stoehr et al., Semi-analytic simulations of galactic winds: volume filling factor, ejection of metals and parameter study, MNRAS 359 (2005) 1201 [astro-ph/0402044].

[16] E. Kado-Fong, J.-G. Kim et al., Diffuse Ionized Gas in Simulations of Multiphase, Star-forming Galactic Disks, ApJ 897 (2020) 143 [2006. 06697].

[17] Y. Sugahara, M. Ouchi et al., Fast Outflows Identified in Early Star-forming Galaxies at $z=$ 5-6, ApJ 886 (2019) 29 [1904.03106].

[18] Y. Inoue, S. Inoue et al., Extragalactic Background Light from Hierarchical Galaxy Formation: Gamma-Ray Attenuation up to the Epoch of Cosmic Reionization and the First Stars, ApJ 768 (2013) 197 [1212 . 1683].

[19] M. Ackermann, M. Ajello et al., The Spectrum of Isotropic Diffuse Gamma-Ray Emission between $100 \mathrm{MeV}$ and $820 \mathrm{GeV}, \mathrm{ApJ} 799$ (2015) 86 [1410 . 3696]. 